\title{
CONCENTRIC SOLID TORI IN THE 3-SPHERE
}

BY

\author{
C. H. EDWARDS, JR.(1)
}

1. Introduction. A torus is the topological product of two circles, while a solid torus is the topological product of a circle and a disk. Two solid tori $B$ and $B^{*}$, with $B \subset$ Int $B^{*}$, are said to be concentric if and only if $\mathrm{Cl}\left(B^{*}-B\right)$ is the topological product of a torus and an interval, while two disjoint tori $T$ and $T^{*}$ in a 3-manifold $M$ are said to be concentric in $M$ if and only if their union is the boundary in $M$ of the product of a torus and an interval. Notice that, for $T$ and $T^{*}$ to be concentric, it suffices for $T$ and $T^{*}$ to be the boundaries of two concentric solid tori $B$ and $B^{*}$ respectively in $M$.

In Theorem 3 the relationship of concentricity for polyhedral solid tori in the 3-sphere $S^{3}$ is characterized by using some results of Schubert [10] to sharpen the concentric toral theorem of Harrold, Griffith, and Posey [9]. Theorem 3 is used to prove that, if $A, B$, and $C$ are tame solid tori in $S^{3}$ with $A \subset$ Int $B$ and $B \subset$ Int $C$, then $A$ and $C$ are concentric if and only if $B$ is concentric with both $A$ and $C$ (Theorem 5). This result implies that, if $\left\{B_{n}\right\}_{1}^{\infty}$ is a sequence of tame solid tori in $S^{3}$ such that $B_{n+1} \subset \operatorname{Int} B_{n}$ for $n \geqq 1$, with $\bigcap_{n-1}^{\infty} B_{n}$ being a tame solid torus $B$, then there is an integer $N$ such that $B_{i}$ and $B_{j}$ are concentric if $i>j \geqq N$. It is shown that the same conclusion follows if $B$ is a tame simple closed curve instead of a tame solid torus, but not if $B$ is a wild simple closed curve. Theorem 5 is used in $\$ 5$ to prove that a closed 3-manifold can not contain an uncountable collection of mutually disjoint tori, no two of which are concentric (Theorem 13).

This paper formed part of a dissertation [6] written at the University of Tennessee under the direction of $O$. G. Harrold, and some of its results are summarized in [7].

2. Pairs of concentric solid tori. The first step towards the determination of a set of conditions, under which two polyhedral solid tori will be concentric, is provided by the following modification of the concentric toral theorem of Harrold, Griffith, and Posey [9].

Theorem 1. Suppose that $B_{1}$ and $B_{2}$ are polyhedral solid tori in $S^{3}$ with boundaries $T_{1}$ and $T_{2}$ respectively such that

(a) $B_{2} C$ Int $B_{1}$.

Presented to the Society, August 30,1960 under the title $S^{3}$ does not contain uncountably many mutually disjoint tori, no two of which are concentric; received by the editors April 2, 1961.

(1) This research was supported in part through the National Science Foundation, Grant 2793. 
(b) There is a polyhedral annular ring $G$ in $\mathrm{Cl}\left(B_{1}-B_{2}\right)$ such that $G \cap T_{i}$ is a simple closed curve $s_{i}$ for $i=1,2$.

(c) There is a pair of disjoint polyhedral disks $D^{a}$ and $D^{b}$ such that $D_{i}^{a}=D^{a} \cap B_{i}$ and $D_{i}^{b}=D^{b} \cap B_{i}$ are disks with boundaries $r_{i}^{a}$ and $r_{i}^{b}$ respectively. Furthermore the 1-cycles $r_{i}^{a}, r_{i}^{b}, s_{i}$ satisfy the homology relations $r_{i}^{a} \times s_{i}, r_{i}^{b} \times s_{i}$ on $T_{i}$, and none is nullhomologous on $T_{i}, i=1,2$. Finally $s_{i}$ meets $r_{i}^{a}$ and $r_{i}^{b}$ each in a single point, $i=1,2$.

(d) $D_{1}^{a} \cap G$ and $D_{1}^{b} \cap G$ are arcs spanning the boundary of $G$ (each has exactly one endpoint on each of the two components of $\mathrm{Bd} G$ ).

Then $B_{1}$ and $B_{2}$ are concentric.

The statement of Theorem 1 differs from the statement of the concentric toral theorem only in that Harrold, Griffith, and Posey assumed $G$ to be a disk rather than an annular ring. Since, however, this disk was used merely to obtain an annular ring with the properties described above, their proof applies here without essential alterations.

By a meridian curve of the polyhedral solid torus $B$ is meant a polygonal simple closed polygon on $\mathrm{Bd} B$ which bounds in $B$ but not in $\mathrm{Bd} B$. A polyhedral disk $D$, such that Int $D \subset$ Int $B$ and such that $\operatorname{Bd} D$ is a meridian curve of $B$, is called a meridianal disk of $B$. By a longitude curve of $B$ is meant a simple closed polygon on $\mathrm{Bd} B$ which is non-nullhomologous in $B$ and which crosses some meridian of $B$ at exactly one point, while a latitude curve of $B$ is a simple closed polygon on $\mathrm{Bd} B$ which is non-nullhomologous in $B$ but nullhomologous in $\mathrm{Cl}\left(S^{3}-B\right)$. It is easily seen that any two meridian curves of $B$ are homologous on $\mathrm{Bd} B$, as are any two latitude curves $[10, \mathrm{p} .161]$, while no meridian curve is homologous on $\mathrm{Bd} B$ to a longitude curve.

Using this terminology, the statement of Theorem 1 can be simplified as follows.

THEOREM 2. Suppose that $B_{1}$ and $B_{2}$ are two polyhedral solid tori in $S^{3}$ with boundaries $T_{1}$ and $T_{2}$ respectively and with $B_{2} \subset$ Int $B_{1}$. If there exist two polyhedral annular rings $R_{1}$ and $R_{2}$ in $\mathrm{Cl}\left(B_{1}-B_{2}\right)$, with $R_{1}$ bounded by two longitude curves on $T_{1}$ and $T_{2}$ respectively, with $R_{2}$ bounded by two meridian curves on $T_{1}$ and $T_{2}$ respectively, and with $R_{1} \cap R_{2}$ being an arc spanning $\mathrm{Bd} R_{1}$, then $B_{1}$ and $B_{2}$ are concentric.

Proof. Define $s_{i}=R_{1} \cap T_{i}$ and $r_{i}^{a}=R_{2} \cap T_{i}$ for $i=1,2$. By hypothesis $r_{2}^{a}$ is a meridian of $B_{2}$ and hence bounds a meridianal disk $D_{2}^{a}$ of $B_{2}$. Then $D^{a}$ $=R_{2} \cup D_{2}^{a}$ is a meridianal disk of $B_{1}$ whose intersection with $B_{2}$ is a meridianal disk of $B_{2}$. It follows from the hypotheses that $s_{i} \times 0, r_{i}^{a} \times 0, s_{i} \times r_{i}^{a}$ on $T_{i}$ for $i=1,2$.

If $D^{b}$ is a meridianal disk of $B_{1}$ sufficiently close to $D^{a}$ but disjoint with $D^{a}$, then $D_{2}^{b}=D^{b} \cap B_{2}$ is a meridianal disk of $B_{2}$, and $D^{b} \cap T_{i}=r_{i}^{b}$ is a simple closed curve not nullhomologous on $T_{i}$ and not homologous to $s_{i}$ on $T_{i}$, and inter- 
secting $s_{i}$ in a single point for $i=1,2$. Then $D^{b} \cap R_{1}$ will be an arc spanning Bd $R_{1}$, just as does $D^{a} \cap R_{1}$.

Theorem 1 now applies directly to imply that $B_{1}$ and $B_{2}$ are concentric.

If $K$ is a polyhedral 3-cell in $S^{3}$, then a chord of $K$ is an oriented polygonal arc $u$ whose endpoints lie on $\mathrm{Bd} K$ but which is otherwise contained in the interior of $K$. If the endpoints of $u$ are joined by a polygonal arc on $\mathrm{Bd} K$, then the knot generated in $K$ by the chord $u$ is defined to be the knot in $S^{3}$ (equivalence class of oriented simple closed polygons under orientation-preserving semilinear autohomeomorphisms of $S^{3}$ ) represented by the polygon $u \cup w$, oriented coherently with $u$. It can be seen that the knot type of $u \cup w$ is independent of the choice of $w \subset \mathrm{Bd} K[10, \mathrm{p} .155]$. If $u \cup w$ is an unknotted simple closed polygon (bounds a disk in $S^{3}$ ), then $u$ is called an $u k n o t t e d$ chord of $K$.

Suppose now that $B$ is a polyhedral solid torus in $S^{3}$. Let $B$ be separated by two disjoint meridianal disks $D_{1}$ and $D_{2}$ into two 3-cells $K_{1}$ and $K_{2}$. Choose two points $x \in \operatorname{Int} D_{1}$ and $y \in \operatorname{Int} D_{2}$. Let $a_{1}$ and $a_{2}$ be unknotted chords of $K_{1}$ and $K_{2}$ respectively joining the points $x$ and $y, a_{1}$ directed from $x$ to $y$ and $a_{2}$ from $y$ to $x$. The oriented simple closed curve $a_{1} \cup a_{2}$ is called an oriented center line of the solid torus $B$. If the orientation of $a=a_{1} \cup a_{2}$ is disregarded, then $a$ is called simply a center line of $B$. It is easily seen that any two center lines $a$ and $a^{\prime}$ of $B$ are equivalent in the sense that there exists a semilinear mapping of $S^{3}$ onto itself, the identity on $S^{3}-B$, carrying $a$ onto $a^{\prime}[10$, p. 158].

If $B$ is a polyhedral solid torus and $k$ is a simple closed polygon interior to $B$, the order of $B$ with respect to $k$ is defined to be the minimal number of points of intersection of a meridianal disk of $B$ with the curve $k$ (for all meridianal disks of $B$ ), and is denoted by $O(B, k)$. If $B$ and $B^{*}$ are polyhedral solid tori with $B C$ Int $B^{*}$, the order of $B^{*}$ with respect to $B$, denoted by $O\left(B^{*}, B\right)$, is defined to be the order of $B^{*}$ with respect to a center line of $B$. This definition of $O\left(B^{*}, B\right)$ is independent of the choice of center line of $B[10$, p. 172]. The two polyhedral solid tori $B$ and $B^{*}$ are said to be equivalently knotted if and only if any two center lines $c$ of $B$ and $c^{*}$ of $B^{*}$ can be so oriented as to represent the same knot in $S^{3}$.

Now suppose that $\bar{k}_{1}$ and $\vec{k}_{2}$ are two arbitrary knots in $S^{3}$. Let $S$ be a polyhedral 2-sphere in $S^{3}$, with $K_{1}$ and $K_{2}$ the closures of its complementary domains. Let $u_{1}$ and $u_{2}$ be oriented chords of $K_{1}$ and $K_{2}$ respectively, with common endpoints $p$ and $q, u_{1}$ directed from $p$ to $q$ and $u_{2}$ from $q$ to $p$, such that $u_{i}$ generates the knot $\vec{k}_{i}$ in $K_{i}, i=1,2$. The knot represented by the oriented polygon $u_{1} \cup u_{2}$ is then defined to be the product $\bar{k}_{1} \bar{k}_{2}$ of the knots $\bar{k}_{1}$ and $\bar{k}_{2}$. This product of knots is associative and commutative, and the knot represented by a plane triangle plays the role of an identity $[10$, p. 156].

A characterization of the relation of concentricity for polyhedral solid tori in $S^{3}$ will now be given. 
ThEOREM 3. Let $B$ and $B^{*}$ be two polyhedral solid tori in $S^{3}$ with $B C$ Int $B^{*}$. Then $B$ and $B^{*}$ are concentric if and only if they are equivalently knotted with $O\left(B^{*}, B\right)=1$.

Proof of sufficiency. The construction here employed is very similar to that used by Schubert in proving that, if $B$ and $B^{*}$ are equivalently knotted with $O\left(B^{*}, B\right)=1$, then there is a semilinear homeomorphism of $S^{3}$ onto itself carrying $B^{*}$ onto $B$ and leaving fixed points outside an arbitrary neighborhood of $\mathrm{Cl}\left(B^{*}-B\right)[10$, p. 178].

Let $T=\mathrm{Bd} B$ and $T^{*}=\mathrm{Bd} B^{*}$. Since $B^{*}$ is of order 1 with respect to $B$, there is a meridianal disk $D^{*}$ of $B^{*}$ which intersects $B$ in a meridianal disk $D$ $\left[10\right.$, p. 174]. Let $E^{*}$ be a second meridianal disk of $B^{*}$ disjoint with $D^{*}$ but sufficiently near $D^{*}$ that $E^{*}$ intersects $B$ in a meridianal disk $E$. Then $D^{*}$ and $E^{*}$ will separate $B^{*}$ into two 3-cells $C_{1}^{*}$ and $C_{2}^{*}$, with $C_{i}^{*}$ bounded by the union of the two disks $D^{*}$ and $E^{*}$ with an annular ring $G_{i}^{*} \subset T^{*}$ for $i=1,2$. Similarly $D$ and $E$ will separate $B$ into two 3 -cells $C_{1} \subset C_{1}^{*}$ and $C_{2} \subset C_{2}^{*}$, with $C_{i}$ bounded by the union of the two disks $D$ and $E$ with an annular ring $G_{i} \subset T$ for $i=1,2$.

Now let $a$ be an oriented center line of the solid torus $B$, with $a=a_{1} \cup a_{2}$, where $a_{1}$ and $a_{2}$ are unknotted chords of the 3-cells $C_{1}$ and $C_{2}$ respectively. Since $O\left(B^{*}, B\right)=1$ by hypothesis, it follows from a theorem of Schubert [10, p. 171] that either $a$ is an oriented center line of $B^{*}$ or $a$ is the product of a knot, different from the identity, with the knot represented by an appropriately oriented center line of $B^{*}$. Because $a$ is a center line of $B$, it represents the same knot as any center line of $B^{*}$, since $B$ and $B^{*}$ are by hypothesis equivalently knotted. It therefore follows that $a$ must be a center line of $B^{*}$ $\left[10\right.$, p. 156]. Therefore $a_{1}$ and $a_{2}$ are unknotted chords of $C_{1}^{*}$ and $C_{2}^{*}$ respectively $[10$, p. 161$]$.

It follows finally that $U_{1}=\mathrm{Cl}\left(C_{1}^{*}-C_{1}\right)$ and $U_{2}=\mathrm{Cl}\left(C_{2}^{*}-C_{2}\right)$ are unknotted solid tori on each of which $d=\mathrm{Bd} D$ and $e=\mathrm{Bd} E$ are latitude curves [11, p. 161]. Then $d^{*}=\mathrm{Bd} D^{*}$ and $e^{*}=\mathrm{Bd} E^{*}$ are also latitude curves on $U_{1}$ and $U_{2}$ because $d$ and $d^{*}$ bound the annular ring $R=\mathrm{Cl}\left(D^{*}-D\right)$ on $\mathrm{Bd} U_{1} \cap \mathrm{Bd} U_{2}$, while $e$ and $e^{*}$ bound the annular ring $S=\mathrm{Cl}\left(E^{*}-E\right)$ on $\mathrm{Bd} U_{1} \cap \mathrm{Bd} U_{2}$.

Now let $m_{1}$ be a meridian curve of $U_{1}$ which intersects each of the curves $d, d^{*}, e, e^{*}$ in exactly one point, and let $M_{1}$ be a meridianal disk of $U_{1}$ bounded by $m_{1}$. Let $m_{2}$ be a meridian curve of $U_{2}$ which intersects each of the latitude curves $d, d^{*}, e, e^{*}$ in exactly one point and such that $m_{1} \cap R=m_{2} \cap R$ and $m_{1} \cap S=m_{2} \cap S$. That such a meridian of $U_{2}$ can be found follows from the elementary observation that, if the boundary $T$ of a solid torus $U$ is separated into two annular rings $A_{1}$ and $A_{2}$ by two latitude curves $n_{1}$ and $n_{2}$, and if $a$ is an arc in $A_{1}$ with one endpoint on $n_{1}$ and the other on $n_{2}$, then there is an $\operatorname{arc} b$ in $A_{2}$ joining the endpoints of $a$ in such a way that $a \cup b$ is a meridian curve of $U$ (this is evident from an examination of the fundamental polygon of a torus). Then let $M_{2}$ be a meridianal disk of $U_{2}$ bounded by $m_{2}$. 
Finally define $R_{1}=\mathrm{Cl}\left(D^{*}-D\right)$ and $R_{2}=M_{1} \cup M_{2}$. It is clear that $R_{1}$ is an annular ring with boundary components $d$ and $d^{*}$ which are meridian curves of $B$ and $B^{*}$ respectively. If $l=\left(m_{1} \cap T\right) \cup\left(m_{2} \cap T\right)$ and $l^{*}=\left(m_{1} \cap T^{*}\right)$ $\cup\left(m_{2} \cap T^{*}\right)$, then clearly $l$ and $l^{*}$ are longitude curves on $T$ and $T^{*}$ respectively (since $m_{1} \cap d=m_{2} \cap d$ and $m_{1} \cap d^{*}=m_{2} \cap d^{*}$ are single points) which together form the boundary of $R_{2}$. Since also $R_{2}$ is the union of the two disks $M_{1}$ and $M_{2}$ intersecting in a pair of arcs common to their boundaries, it follows that $R_{2}$ is an annular ring.

Since the annular rings $R_{1}$ and $R_{2}$ are both contained in $U_{1} \cup U_{2}$ $=\mathrm{Cl}\left(B^{*}-B\right)$, and since $R_{1} \cap R_{2}$ is the arc $M_{1} \cap R=M_{2} \cap R$ spanning the boundaries of $R_{1}$ and $R_{2}$, it follows from Theorem 2 that $B^{*}$ and $B$ are concentric.

Proof of necessity. Suppose now that $B$ and $B^{*}$ are concentric. Then $\mathrm{Cl}\left(B^{*}-B\right)$ can be regarded as the topological product of $T$ and the closed unit interval $[0,1]$ with $T \times\{0\}=T$ and $T \times\{1\}=T^{*}$. Since $B$ and $B^{*}$ are polyhedral, it may be assumed that if $K \subset T$ is polyhedral, then $K \times[0,1]$ is polyhedral.

Let $m_{1}$ and $m_{2}$ be two meridian curves on $T$ bounding disjoint meridianal disks $D_{1}$ and $D_{2}$ respectively of $B$. Define $R_{i}$ to be the annular ring $m_{i} \times[0,1]$ for $i=1,2$. Then $D_{1}^{*}=D_{1} \cup R_{1}$ and $D_{2}^{*}=D_{2} \cup R_{2}$ are disks whose interiors are contained in Int $B^{*}$ and whose boundaries are the simple closed curves $m_{1}^{*}=m_{1} \times\{1\}$ and $m_{2}^{*}=m_{2} \times\{1\}$ respectively on $T^{*}$. Since the projection mapping $p \times\{0\} \rightarrow p \times\{1\}$, being a homeomorphism carrying the torus $T$ onto the torus $T^{*}$, carries non-nullhomologous curves on $T$ onto non-nullhomologous curves on $T^{*}$, it follows that $m_{1}^{*}$ and $m_{2}^{*}$ are not nullhomologous on $T^{*}$. Consequently $D_{1}^{*}$ and $D_{2}^{*}$ are disjoint meridianal disks of $B^{*}$ intersecting $B$ in the meridianal disks $D_{1}$ and $D_{2}$ respectively.

Hence $D_{1}$ and $D_{2}$ separate $B$ into two closed 3-cells $K_{1}$ and $K_{2}$ respectively, while $D_{1}^{*}$ and $D_{2}^{*}$ separate $B^{*}$ into two closed 3-cells $K_{1}^{*}$ and $K_{2}^{*}$ respectively. Let the subscripts be chosen in such a manner that $K_{1} \subset K_{1}^{*}$ and $K_{2} \subset K_{2}^{*}$.

Now let $u$ be a center line of $B$. which intersects the 3-cells $K_{1}$ and $K_{2}$ in the unknotted chords $u_{1}$ and $u_{2}$ respectively. Then $u_{1}$ and $u_{2}$ are chords of $K_{1}^{*}$ and $K_{2}^{*}$ respectively, and to show that $u$ is also a center line of $B^{*}$, it suffices to show that $u_{1}$ and $u_{2}$ are unknotted in $K_{1}^{*}$ and $K_{2}^{*}$ respectively.

But since $\mathrm{Cl}\left(K_{i}^{*}-K_{i}\right)$ is the solid torus $G_{i} \times[0,1]$, where $G_{1}$ and $G_{2}$ are the two annular rings into which $m_{1}$ and $m_{2}$ separate $T$, it follows $[10$, p. 167] that $u_{i}$ generates the same knot in $K_{i}$ and $K_{i}^{*}$ for $i=1,2$. Thus $u_{1}$ and $u_{2}$ are unknotted chords of $K_{1}^{*}$ and $K_{2}^{*}$ respectively, so that $u$ is a center line of $B^{*}$. But the fact that $B$ and $B^{*}$ share the common center line $u$ implies immediately that $B$ and $B^{*}$ are equivalently knotted with $O\left(B^{*}, B\right)=1$. This completes the proof of Theorem 3 .

Lemma 1. Suppose that $B$ and $B^{*}$ are polyhedral solid tori in $S^{3}$ with $B C$ Int $B^{*}$. Then $O\left(B^{*}, B\right)=1$ if and only if there is a polyhedral annular ring 
$R$ in $\mathrm{Cl}\left(B^{*}-B\right)$ bounded by two meridian curves $m$ and $m^{*}$ on $B$ and $B^{*}$ respectively.

Proof. Suppose that $O\left(B^{*}, B\right)=1$. Then there is a meridianal disk $D^{*}$ of $B^{*}$ such that $D=D^{*} \cap B$ is a meridianal disk of $B$. Thus $R=\operatorname{Cl}\left(D^{*}-D\right)$ is an annular ring in $\mathrm{Cl}\left(B^{*}-B\right)$ bounded by two meridian curves on $B$ and $B^{*}$ respectively.

To prove the converse, assume the existence of the annular ring $R$. Let $k$ be a center line of $B$, and let $D$ be a meridianal disk of $B$ which intersects $k$ in a single point and whose boundary is $m$. Then, since the meridianal disk $D^{*}=D \cup R$ of $B^{*}$ intersects the center line $k$ of $B$ in a single point, it follows that $O\left(B^{*}, B\right)$ is either 0 or 1 . If $O\left(B^{*}, B\right)=0$, then the algebraic intersection number of $D^{*}$ with $k$ (to obtain the algebraic intersection number of a polyhedral disk with a polygonal line in general position, associate with each of the points of intersection the number +1 or -1 according to the sense of piercing, and compute the sum of the numbers thus defined) must be $0[10$, p. 170], so that $D^{*}$ must intersect $k$ in an even number of points. This contradiction proves that $O\left(B^{*}, B\right)=1$.

Lemma 2. Suppose that $B$ and $B^{*}$ are polyhedral solid tori in $S^{8}$ with $B C$ Int $B^{*}$. If there is a polyhedral annular ring $R$ in $\mathrm{Cl}\left(B^{*}-B\right)$ bounded by two longitude curves on $B$ and $B^{*}$ respectively, then $B$ and $B^{*}$ are equivalently knotted.

Proof. This follows immediately from the fact that the two boundary curves of an annular ring in $S^{3}$ are equivalent and the fact that a center line and a longitude curve of a polyhedral solid torus are equivalent.

Theorem 4. Let $B$ and $B^{*}$ be two polyhedral solid tori in $S^{3}$ with $B C$ Int $B^{*}$. Consider the following conditions:

(a) $O\left(B^{*}, B\right)=1$.

(b) There is a polyhedral annular ring $R$ in $\mathrm{Cl}\left(B^{*}-B\right)$ bounded by two meridian curves on $B$ and $B^{*}$ respectively.

(c) $B$ and $B^{*}$ are equivalently knotted.

(d) There is a polyhedral annular ring $R$ in $\mathrm{Cl}\left(B^{*}-B\right)$ bounded by two longitude curves on $B$ and $B^{*}$ respectively.

If either of the first two conditions holds simultaneously with either of the last two conditions, then $B$ and $B^{*}$ are concentric.

Proof. By Lemma 1, (b) implies (a). By Lemma 2, (d) implies (c). But, by Theorem 3, (a) and (c) suffice to imply that $B$ and $B^{*}$ are concentric.

Corollary 1. Suppose that $B$ and $B^{*}$ are unknotted polyhedral solid tori in $S^{3}$ with $B C$ Int $B^{*}$. If there is a polyhedral annular ring $R$ in $\mathrm{Cl}\left(B^{*}-B\right)$ 
bounded by two meridian curves on $B$ and $B^{*}$ respectively, then $B$ and $B^{*}$ are concentric.

Proof. Lemma 1 implies that $O\left(B^{*}, B\right)=1$. But $B$ and $B^{*}$ are equivalently knotted, both being unknotted. Theorem 3 then shows that $B$ and $B^{*}$ are concentric.

Corollary 2. Suppose that $B$ and $B^{*}$ are knotted polyhedral solid tori in $S^{3}, B$ interior to $B^{*}$, which are equivalently knotted. If there is a polyhedral annular ring $R$ in $\mathrm{Cl}\left(B^{*}-B\right)$ whose boundary components $m$ and $m^{*}$ are unknotted non-nullhomologous simple closed curves on $\mathrm{Bd} B$ and $\mathrm{Bd} B^{*}$ respectively, then $B$ and $B^{*}$ are concentric.

Proof. Since an unknotted simple closed curve on the boundary of a knotted solid torus is either nullhomologous or is a meridian curve $[10$, p. 164], it follows that $m$ is a meridian curve of $B$, and $m^{*}$ is a meridian curve of $B^{*}$. Lemma 1 then applies to show that $O\left(B^{*}, B\right)=1$. Since $B$ and $B^{*}$ are equivalently knotted by hypothesis, Theorem 3 implies that $B$ and $B^{*}$ are concentric.

3. Sequences of concentric solid tori. The following theorem is one of the principal tools to be used in studying infinite collections of solid tori.

Theorem 5. Suppose that $A, B$, and $C$ are tame solid tori in $S^{3}$ with $A \subset$ Int $B$ and $B C$ Int $C$. Then $A$ and $C$ are concentric if and only if $B$ is concentric with both $A$ and $C$.

Proof. A compact subset of $S^{3}$ is said to be tame if it can be carried onto a polyhedral set by a homeomorphism of $S^{3}$ onto itself. Since there is therefore a homeomorphism of $S^{3}$ onto itself carrying $A, B$, and $C$ onto polyhedral solid tori, it may be assumed without loss that $A, B$, and $C$ are themselves polyhedral.

Suppose first that $A$ and $C$ are concentric. It then follows from Theorem 3 that $A$ and $C$ are equivalently knotted with $O(C, A)=1$. Schubert has shown that, if $k$ is a simple closed polygon interior to the polyhedral solid torus $B_{1}$, with $B_{1}$ interior to the polyhedral solid torus $B_{2}$, then $O\left(B_{2}, k\right)=O\left(B_{2}, B_{1}\right)$ $\cdot O\left(B_{1}, k\right)[10$, p. 175]. Therefore $O(C, B) \cdot O(B, A)=O(C, A)=1$, so that $O(C, B)=O(B, A)=1$, since the order of one solid torus with respect to another is a non-negative integer.

It remains to be proved that $A, B$, and $C$ are equivalently knotted. Since it was shown in the second part of the proof of Theorem 3 that any two concentric polyhedral solid tori have a common center line, let $a$ be an oriented polygon interior to $A$ which is an oriented center line of both $A$ and $C$. Let $b$ be a center line of $B$, oriented positively with respect to $a$, that is, so that $a$ is homologous in $B$ to a positive multiple of $b$, the generator of the 1-dimensional homology group of $B$. Since $O(B, a)=1$, it follows that $a \sim b$ in $B$ 
$[10$, p. 171$]$, and hence also that $a \sim b$ in $C$. Now Schubert has shown that, if $D$ is a polyhedral solid torus in $S^{3}$ with oriented center line $d$ and if $k$ is an oriented polygon interior to $D$ with $O(D, k)=1$ and $d \sim k$ in $D$, then there is a knot $\bar{z}$ such that $\bar{k}=\bar{d} \bar{z}$, where $\bar{k}$ and $\bar{d}$ are the knots represented by $k$ and $d$ respectively $\left[10\right.$, p. 171]. Denoting by $\bar{a}$ and $b$ the knots in $S^{3}$ represented by $a$ and $b$ respectively, it follows that there is a knot $\bar{x}$ such that $\bar{a}=\bar{b} \bar{x}$, because $a \subset$ Int $B$ with $O(B, a)=1$. Since $b \subset$ Int $C$ with $O(C, b)=1$, it follows that there is a knot $\bar{y}$ such that $\bar{b}=\bar{a} \bar{y}$. Substitution then gives $\bar{b}=\bar{b} \bar{x} \bar{y}$. But Schubert has also shown that the genus of a product knot is equal to the sum of the genera of its factors, and that the identity knot is the only knot whose genus is zero [11]. Thus the genus of each of $\bar{x}$ and $\bar{y}$ is zero, so that each of $\bar{x}$ and $\bar{y}$ is the identity knot.

Consequently $\bar{a}=\bar{b}$, so that the polyhedral solid tori $A, B$, and $C$ are equivalently knotted. It now follows from Theorem 3 that $B$ is concentric with both $A$ and $C$.

To prove the converse, suppose that $B$ is concentric with both $A$ and $C$. Then, by Theorem $3, A$ and $C$ are equivalently knotted with $O(C, A)$ $=O(C, B) \cdot O(B, A)=1$, so that $A$ and $C$ are concentric.

Lemma 3. If $B$ is a polyhedral solid torus in $S^{3}$, then there exist two polyhedral solid tori $B_{1}$ and $B_{2}$, each concentric with $B$, such that $B_{1} \subset \operatorname{Int} B$ and $B \subset$ Int $B_{2}$.

Proof. Let $b$ be a center line of the solid torus $B$, and let $k$ be a polygonal closed curve in $S^{3}$ equivalent to $b$. Since, given any curve $k$, there is in each neighborhood of $k$ a polyhedral solid torus having $k$ as a center line $[10$, p. 177], choose a polyhedral solid torus $C_{2}$ with $k$ as center line. Then let $C$ be a polyhedral solid torus contained in Int $C_{2}$ with $k$ as a center line, and let $C_{1}$ be a polyhedral solid torus contained in Int $C$ with $k$ as center line.

Since $B$ and $C$ are polyhedral solid tori having equivalent center lines $b$ and $k$ respectively, there is a semilinear homeomorphism $f$ of $S^{3}$ onto itself which maps $C$ onto $B$ and $k$ onto $b\left[10\right.$, p. 180]. If $B_{i}=f\left(C_{i}\right)$ for $i=1,2$, then clearly $B_{1} \subset$ Int $B$ and $B C$ Int $B_{2}$. Since $B, B_{1}$, and $B_{2}$ share the common center line $b$ (center lines are preserved under semilinear maps of solid tori), it follows from Theorem 3 that they are mutually concentric.

Corollary 3. Suppose that $\left\{B_{n}\right\}_{1}^{\infty}$ is a sequence of tame solid tori in $S^{3}$, with $B_{n+1} \subset$ Int $B_{n}$ for $n \geqq 1$, such that $A=\bigcap_{n=1}^{\infty} B_{n}$ is a tame solid torus. Then there exists an integer $N$ such that $B_{i}$ and $B_{j}$ are concentric with each other and with $A$ if $i>j \geqq N$.

Proof. Using Lemma 3, choose a tame solid torus $C$ such that $A \subset$ Int $C$ with $A$ and $C$ concentric. Since $\left\{S^{3}-B_{n}\right\}_{1}^{\infty}$ is an increasing sequence of open sets whose union is $S^{3}-A$, and which therefore covers the compact set $S^{3}-$ Int $C$, there exists an integer $N$ such that $S^{3}-\operatorname{Int} C \subset S^{3}-B_{j}$, or $B_{j} \subset$ Int $C$, whenever $j \geqq N$. It then follows from Theorem 5 that $A$ and $B_{j}$ 
are concentric for $j \geqq N$. If $i>j \geqq N, A$ is interior to $B_{i}$ and $B_{i}$ is interior to $B_{j}$, so that Theorem 5 implies that $B_{i}$ is concentric with both $A$ and $B_{j}$.

Corollary 4. Suppose that $\left\{B_{n}\right\}_{1}^{\infty}$ is a sequence of tame solid tori in $S^{3}$, with $B_{n} \subset$ Int $B_{n+1}$ for $n \geqq 1$, such that $\bigcup_{n=1}^{\infty} B_{n}$ is the interior of a tame solid torus $A$. Then there exists an integer $N$ such that $B_{i}$ and $B_{j}$ are concentric with each other and with $A$ if $i>j \geqq N$.

The proof of Corollary 4 is similar to that of Corollary 3.

Theorem 6. Suppose that $K$ is a tame simple closed curve in $S^{3}$ and that $\left\{B_{n}\right\}_{1}^{\infty}$ is a sequence of tame solid tori, with $B_{n+1} \subset \operatorname{Int} B_{n}$ for $n \geqq 1$, such that $\bigcap_{n=1}^{\infty} B_{n}=K$. Then there is an integer $N$ such that $B_{i}$ and $B_{j}$ are concentric if $i>j \geqq N$.

Proof. Since there is a homeomorphism of $S^{3}$ onto itself carrying $K$ onto a simple closed polygon, it may be assumed that $K$ itself is a polygonal simple closed curve. Then there is a polyhedral solid torus $C$ having $K$ as a center line $\left[10\right.$, p. 177]. Since $\left\{S^{3}-B_{n}\right\}_{1}^{\infty}$ is an increasing sequence of open sets covering the compact set $S^{3}-$ Int $C$, there is an integer $N$ such that $B_{j} \subset \operatorname{Int} C$ if $j \geqq N$. Given $i$ and $j$ such that $i>j \geqq N$, choose a polyhedral solid torus $A$ interior to $B_{i}$ having $K$ as a center line $[10$, p. 177]. Since $A$ and $C$ have the same center line, it follows from Theorem 3 that they are concentric. Then Theorem 5 implies that each of $B_{i}$ and $B_{j}$ is concentric with both $A$ and $C$, and another application of Theorem 5 shows that $B_{i}$ and $B_{j}$ are concentric with each other.

Corollary 5. Suppose that $\left\{B_{n}\right\}_{1}^{\infty}$ is a sequence of tame solid tori in $S^{3}$, with $B_{n+1} \subset$ Int $B_{n}$ for $n \geqq 1$, such that no two of the tori $\left\{B_{n}\right\}_{1}^{\infty}$ are concentric. If $\bigcap_{n-1}^{\infty} B_{n}$ is a simple closed curve $K$, then $K$ is wildly imbedded in $S^{3}$.

EXAmple. In order to see the necessity of the hypothesis in Theorem 6 that the simple closed curve $K$ is tame, that is, that the situation described in Corollary 5 can actually occur, consider a simple closed curve $C$ constructed as follows: Let $J$ be the boundary of the square in the $x y$-plane (in $E^{3}$ compactified at infinity) whose vertices are the points $(1,1,0),(-1,1,0)$, $(-1,-1,0)$, and $(1,-1,0)$. Denote by $x_{n}$ the point $(1 / n, 0,0)$ on $J$. For each positive integer $n \geqq 1$ let $K_{n}$ be the 3-cell consisting of all points of $E^{3}$ whose distance from $x_{n}$ is not greater than $1 / 3 n(n+1)$. Then the 3-cells $\left\{K_{n}\right\}_{1}^{\infty}$ are mutually disjoint, and the intersection of $K_{n}$ with $J$ is a segment $w_{n}$ with endpoints $p_{n}$ and $q_{n}$. For each $n$ let $u_{n}$ be a polygonal chord of $K_{n}$, with endpoints $p_{n}$ and $q_{n}$, such that $u_{n}$ generates a trefoil knot in $K_{n}$. The simple closed curve $C$ is now defined by

$$
C=\left(J-\bigcup_{n=1}^{\infty} w_{n}\right) \cup \bigcup_{n=1}^{\infty} u_{n} .
$$


It is clear that $C$ can be expressed as the intersection of a decreasing sequence $\left\{B_{i}\right\}_{1}^{\infty}$ of tame solid tori, such that the solid torus $B_{i}$ follows $C$ through the first $i$ 3-cells $K_{1}, K_{2}, \cdots, K_{i}$, but contains $\bigcup_{n=i+1}^{\infty} K_{n}$ in its interior, so that the simple closed polygon $C_{i}=\left(J-\bigcup_{n=1}^{i} w_{n}\right) \cup \bigcup_{n=1}^{i} u_{n}$ is a center line of $B_{i}$. Since $C_{i}$ is then the knot product of $i$ trefoil knots, no two of the tame solid tori $\left\{B_{i}\right\}_{1}^{\infty}$ are equivalently knotted, so that by Theorem 3 no two are concentric.

The property of tame curves in $S^{3}$ which is suggested by Theorem 6 and the above example has been used to give a new characterization of tame simple closed curves in the 3-sphere [8].

TheOREM 7. Suppose that $\left\{B_{n}\right\}_{1}^{\infty}$ and $\left\{C_{n}\right\}_{1}^{\infty}$ are two sequences of tame solid tori in $S^{3}$, with $B_{n+1} \subset$ Int $B_{n}$ and $C_{n+1} \subset$ Int $C_{n}$ for $n \geqq 1$, such that $\bigcap_{n=1}^{\infty} B_{n}$ $=\bigcap_{n=1}^{\infty} C_{n}$. If there is an integer $N$ such that $B_{i}$ and $B_{j}$ are concentric when $i>j$ $\geqq N$, then there exists an integer $M$ such that $C_{i}$ and $C_{j}$ are concentric when $i>j \geqq M$.

Proof. If $K=\bigcap_{n=1}^{\infty} B_{n}=\bigcap_{n=1}^{\infty} C_{n}$, then $S^{3}-$ Int $B_{N} \subset S^{3}-K=\bigcup_{n=1}^{\infty}\left(S^{3}-C_{n}\right)$ so that $\left\{S^{3}-C_{n}\right\}_{1}^{\infty}$ is an increasing sequence of open sets covering the compact set $S^{3}-\operatorname{lnt} B_{N}$. It follows that there is an integer $M$ such that $C_{j} \subset \operatorname{Int} B_{N}$ if $j \geqq M$. Now let $i$ and $j$ be two positive integers such that $i>j \geqq M$. Since Int $C_{i}$ is a neighborhood of $K$, there is an integer $P$ such that $B_{P} \subset \operatorname{Int} C_{i}$. Clearly $P>N$ so that $B_{P}$ and $B_{N}$ are concentric. It now follows from Theorem 5 that $C_{i}$ and $C_{j}$ are both concentric with $B_{N}$, and another application of Theorem 5 shows that $C_{i}$ and $C_{j}$ are concentric with each other.

The following theorem and its proof illustrate a useful technique for replacing a sequence of concentric tame solid tori with a sequence of concentric polyhedral solid tori.

THEOREM 8. If $\left\{B_{n}\right\}_{1}^{\infty}$ is a sequence of tame solid tori in $S^{3}$ with $B_{n+1}$ $C$ Int $B_{n}$ for $n \geqq 1$, then there is a homeomorphism $f$ of $S^{3}$ onto itself such that (a) $f(x)=x$ if $x \in K=\bigcap_{n=1}^{\infty} B_{n}$, and (b) each of the solid tori $\left\{f\left(B_{n}\right)\right\}_{1}^{\infty}$ is polyhedral.

Proof. For each $n \geqq 1$, denote by $T_{n}$ the tame torus bounding the solid torus $B_{n}$, and define

$$
\epsilon_{n}=\min \left\{\frac{1}{n}, \frac{1}{3} \rho\left(T_{n}, T_{n-1}\right), \frac{1}{3} \rho\left(T_{n}, T_{n+1}\right)\right\},
$$

where $\rho$ is the Euclidean metric in $S^{3}$. If $U_{n}=\left\{x \in S^{3}: \rho\left(x, T_{n}\right)<\epsilon_{n}\right\}$ for each $n \geqq 1$, then clearly $U_{m} \cap U_{n}=\square$ whenever $m \neq n$. For each $n$ let $f_{n}$ be a homeomorphism of $S^{3}$ onto itself which (a) carries $T_{n}$ onto a polyhedron, (b) moves each point a distance less than $\epsilon_{n}$, and (c) is the identity on $S^{3}-U_{n}$. The existence of such homeomorphisms is given by Theorem 9 of [3], which states that, if $K$ is a locally tame closed subset of a triangulated 3-manifold 
$M$ with boundary (possibly vacuous), and if $C$ is a closed subset of $M$ such that $K$ is locally polyhedral at each point of $K \cap C$, with $\phi$ a positive continuous function on $M-C$, then there exists a homeomorphism $g$ of $M$ onto itself such that $x=g(x)$ if $x \in C, g(K)$ is a polyhedron and $\rho(x, g(x))<\phi(x)$ if $x \in M-C$. Now define a map $f$ on $S^{3}$ by

$$
f(x)= \begin{cases}x & \text { if } x \in S^{3}-\bigcup_{n=1}^{\infty} U_{n}, \\ f_{n}(x) & \text { if } x \in U_{n} .\end{cases}
$$

Since the identity is $1-1$ on $S^{3}-U_{n=1}^{\infty} U_{n}$, and since $f_{n}$ is a homeomorphism of $U_{n}$ onto itself, it is clear that $f$ is a 1-1 mapping of $S^{3}$ onto itself. It is also clear that $f$ is continuous at each point of $S^{3}-K$, since $f$ agrees with the identity in some neighborhood of each point of $S^{3}-K-U_{n=1}^{\infty} \mathrm{Cl} U_{n}$, and with $f_{n}$ in some neighborhood of a point of $U_{n}$, while $f_{n}$ matches with the identity in a continuous manner at each point of $\mathrm{Bd} U_{n}$.

To show that $f$ is a homeomorphism of $S^{3}$ onto itself, it therefore suffices to show that $f$ is continuous at each point of $K$, because $S^{3}$ is compact and Hausdorff. Since $f$ is the identity on the closed set $K$ it hence suffices to show that if $\left\{x_{k}\right\}_{1}^{\infty}$ is a sequence of points of $S^{3}-K$ converging to a point $x$ of $\operatorname{Bd} K$, then the sequence $\left\{f\left(x_{k}\right)\right\}_{1}^{\infty}$ converges to $x$. Since $f\left(x_{k}\right)=x_{k}$ if $x_{k} \in S^{3}$ $-U_{n=1}^{\infty} U_{n}$, it may be assumed without loss that each point $x_{k}$ of the sequence lies in one of the sets $U_{n}$, say $U_{n_{k}}$, so that $\rho\left(x_{k}, f\left(x_{k}\right)\right)=\rho\left(x_{k}, f_{n_{k}}\left(x_{k}\right)\right)<\epsilon_{n_{k}}$ for each $k \geqq 1$. It is clear that $\lim _{k \rightarrow \infty} n_{k}=\infty$. For otherwise, if there were an integer $N$ such that $n_{k}<N$ for infinitely many values of $k$, then infinitely many points of the sequence $\left\{x_{k}\right\}_{1}^{\infty}$ would lie in $S^{3}-B_{N}$, which would contradict the fact that $\left\{x_{k}\right\}_{1}^{\infty}$ converges to $x \in K$, since $B_{N}$ contains $K$ in its interior. It follows that $\lim _{k \rightarrow \infty} \rho\left(x_{k}, f\left(x_{k}\right)\right) \leqq \lim _{k \rightarrow \infty} \epsilon_{n_{k}} \leqq \lim _{k \rightarrow \infty} 1 / n_{k}=0$. Consequently $\lim _{k \rightarrow \infty} f\left(x_{k}\right)=\lim _{k \rightarrow \infty} x_{k}=x=f(x)$ so that $f$ is continuous at $x$. Therefore $f$ is a homeomorphism of $S^{3}$ onto itself which is the identity on $K$ and carries each of the solid tori $\left\{B_{n}\right\}_{1}^{\infty}$ onto a polyhedron.

4. Similarly situated solid tori. Two solid tori $B_{1}$ and $B_{2}$ in $S^{3}$ are said to be similarly situated if and only if there exist two concentric tame solid tori $A_{1}$ and $A_{2}$ in $S^{3}$ such that $A_{1}$ is interior to both $B_{1}$ and $B_{2}$, and $B_{1}$ and $B_{2}$ are both interior to $A_{2}$.

TheOREM 9. Two tame solid tori $B_{1}$ and $B_{2}$ in $S^{3}$ are concentric if and only if they are similarly situated and have disjoint boundaries.

Proof. First suppose that $B_{1}$ and $B_{2}$ are similarly situated and have disjoint boundaries $T_{1}$ and $T_{2}$. Then by definition there are two concentric tame solid tori $A_{1}$ and $A_{2}$ such that $A_{1} \subset$ Int $B_{1} \cap$ Int $B_{2}$ and $B_{1} \cup B_{2} \subset$ Int $A_{2}$. It follows that either $B_{1} \subset$ Int $B_{2}$ or $B_{2} \subset$ Int $B_{1}$. To see this, choose $x \in \operatorname{Int} A_{1}$ and $y \in S^{3}-A_{2}$ and suppose that $T_{1} \subset \operatorname{Int} B_{2}$. If there were a point $z \in \operatorname{Int} B_{1}$ 
- Int $B_{2}, x$ could be joined to $z$ by an arc in Int $B_{1}$, and $y$ could be joined to $z$ by an arc in $S^{3}$-Int $B_{2}$ not intersecting $T_{1}$, which contradicts the fact that $T_{1}$ separates $x$ and $y$ in $S^{3}$. Hence $T_{1} \subset$ Int $B_{2}$ implies $B_{1} \subset$ Int $B_{2}$. Since $T_{1} \cap T_{2}=\square$, the only other possibility is $T_{1} \subset S^{3}-B_{2}$, which implies similarly that $B_{2} \subset$ Int $B_{1}$. Therefore Theorem 5 may be applied to show that $B_{1}$ and $B_{2}$ are concentric.

Conversely, if $B_{1}$ and $B_{2}$ are concentric with $B_{1} \subset$ Int $B_{2}$, for instance, Lemma 3 can be used to find tame solid tori $A_{1}$ and $A_{2}$, with $A_{1}$ interior to and concentric with $B_{1}$, and with $B_{2}$ interior to and concentric with $A_{2}$. Theorem 5 then implies that $A_{1}$ and $A_{2}$ are concentric, so that $B_{1}$ and $B_{2}$ are similarly situated with disjoint boundaries.

Suppose that $\left\{B_{n}\right\}_{0}^{\infty}$ is a sequence of homeomorphic compact sets in $S^{3}$. It is said that the sequence $\left\{B_{n}\right\}_{1}^{\infty}$ converges homeomorphically to $B_{0}$ if and only if there exists a sequence $\left\{\epsilon_{n}\right\}_{1}^{\infty}$ of positive numbers, converging to zero, and a sequence $\left\{h_{n}\right\}_{1}^{\infty}$ of homeomorphisms, with $h_{n}$ carrying $B_{n}$ onto $B_{0}$ in such a way that $\rho\left(x, h_{n}(x)\right) \leqq \epsilon_{n}$ for every $x \in B_{n}$, where $\rho$ is the standard metric for $S^{3}$.

THEOREM 10. Suppose that $\left\{B_{n}\right\}_{1}^{\infty}$ is a sequence of solid tori in $S^{3}$ converging homeomorphically to the tame solid torus $B$. Then there exists an integer $N$ such that $B_{n}$ and $B$ are similarly situated for $n \geqq N$.

Proof. By Lemma 3 choose two tame solid tori $A_{1}$ and $A_{2}$, each concentric with $B$, with $A_{1}$ interior to $B$ and $B$ interior to $A_{2}$. Define

$$
\alpha_{1}=\min \left\{\rho\left(\mathrm{Bd} B, \mathrm{Bd} A_{1}\right), \rho\left(\operatorname{Bd} B, \operatorname{Bd} A_{2}\right)\right\} \text {. }
$$

Then choose a fixed interior point $p_{1}$ of $A_{1}$, a fixed point $p_{2}$ of $S^{3}-A_{2}$, and define $\alpha=\min \left\{\alpha_{1}, \rho\left(p_{1}, \operatorname{Bd} A_{1}\right), \rho\left(p_{2}, \operatorname{Bd} A_{2}\right)\right\}$. Now let $\left\{h_{n}\right\}_{1}^{\infty}$ be a sequence of homeomorphisms, $h_{n}$ taking $B_{n}$ onto $B$ with $\max _{x \in B_{n}} \rho\left[x, h_{n}(x)\right] \leqq \epsilon_{n}$, where $\left\{\epsilon_{n}\right\}_{1}^{\infty}$ is a sequence of positive numbers converging to zero. Choose an integer $N$ such that $\epsilon_{n}<\alpha$ if $n \geqq N$.

If $n \geqq N$ and $y \in \mathrm{Bd} B_{n}$, it then follows that $\rho(y, \operatorname{Bd} B) \leqq \rho\left(y, h_{n}(y)\right) \leqq \epsilon_{n}<\alpha$ so that $\rho(y, \operatorname{Bd} B)<\alpha_{1}$. Therefore $\mathrm{Bd} B_{n}$ is exterior to $A_{1}$ and interior to $A_{2}$. Either $A_{1} \subset$ Int $B_{n}$ or $B_{n} \subset S^{3}-A_{1}$. To the contrary, suppose that there exist points $x \in A_{1}-B_{n}$ and $y \in B_{n}-\left(S^{3}-A_{1}\right)=B_{n} \cap A_{1}$. Then $x$ and $y$ can be joined by an arc in $A_{1}$ not intersecting $\mathrm{Bd} B_{n}$, which contradicts the fact that $\mathrm{Bd} B_{n}$ separates $x$ and $y$ in $S^{3}$. Now suppose that $B_{n} \subset S^{3}-A_{1}$. Since $p_{1} \in A_{1} \subset B$, there must exist a point $q \in B_{n}$ such that $h_{n}(q)=p_{1}$. But this implies that $\rho\left(q, h_{n}(q)\right)=\rho\left(q, p_{1}\right) \geqq\left(p_{1}, \mathrm{Bd} A_{1}\right) \geqq \alpha>\epsilon_{n}$ which is a contradiction. Therefore $A_{1} \subset$ Int $B_{n}$. It may be shown similarly that $B_{n} \subset$ Int $A_{2}$.

Since $A_{1}$ and $A_{2}$ are concentric by Theorem 5 , it follows that $B$ and $B_{n}$ are similarly situated if $n \geqq N$, so that the proof of Theorem 10 is complete.

It can be shown that if $s$ is a simple closed polygon contained in the intersection of the boundaries of two similarly situated polyhedral solid tori $B_{1}$ 
and $B_{2}$, then $s$ is nullhomologous on $B d B_{1}$ if and only if it is nullhomologous on $\mathrm{Bd} B_{2}\left[6\right.$, p. 48]. This result can be used to prove that, if $B_{1}$ and $B_{2}$ are similarly situated polyhedral solid tori, with $A_{1}$ and $A_{2}$ two concentric polyhedral solid tori such that $A_{1} \subset$ Int $B_{1} \cap$ Int $B_{2}$ and $B_{1} \cup B_{2} \subset$ Int $A_{2}$, then there is a semilinear homeomorphism of $S^{3}$ onto itself which carries $B_{1}$ onto $B_{2}$ and is the identity on $A_{1} \cup\left(S^{3}-A_{2}\right)[6$, p. 50].

5. Uncountable collections of concentric tori( $\left.{ }^{2}\right)$. If $A$ is a subset of the space $X$, then $A$ is said to be collared in $X$ if and only if there is a homeomorphism $f$ of $A \times[-1,1]$ into $X$ such that $f(a, 0)=a$ for each $a \in A$. Then $f(A \times[-1,1])$ is called a collar of $A$ in $X$.

\section{Lemma 4. If $S$ is a collared closed 2-manifold in $S^{3}$, then $S$ is tame in $S^{3}$.}

Proof. Let $f$ be a homeomorphism of $S \times[-1,1]$ into $S^{3}$ such that $f(x, 0)=x$ for each $x \in S$. For each positive integer $n$, define $S_{n}^{\prime}=f(S \times\{-1 / n\})$ and $S_{n}^{\prime \prime}=f(S \times\{1 / n\})$. Let the homeomorphisms $h_{n}^{\prime}$ and $h_{n}^{\prime \prime}$ of $S$ onto $S_{n}^{\prime}$ and $S_{n}^{\prime \prime}$ respectively be defined by $h_{n}^{\prime}(x)=f(x,-1 / n)$ and $h_{n}^{\prime \prime}(x)=f(x, 1 / n)$ for each $x \in S, n=1,2, \cdots$. Then $\lim _{n \rightarrow \infty} \max _{x \in S} \rho\left[x, h_{n}^{\prime}(x)\right]=0$. To the contrary, suppose that there is an $\epsilon>0$, an increasing sequence $\left\{n_{k}\right\}_{k=1}^{\infty}$ of positive integers, and a sequence $\left\{x_{k}\right\}_{1}^{\infty}$ of points of $S$ such that $\rho\left[x_{k}, h_{n_{k}}^{\prime}\left(x_{k}\right)\right]$ $\geqq \epsilon>0$ for each $k=1,2, \cdots$. Since $S$ is compact it may be assumed that the sequence $\left\{x_{k}\right\}_{1}^{\infty}$ converges to a point $x_{0} \in S$. But then $\lim _{k \rightarrow \infty} \rho\left[x_{k}, h_{n_{k}}^{\prime}\left(x_{k}\right)\right]$ $=\lim _{k \rightarrow \infty} \rho\left[x_{k}, f\left(x_{k},-1 / n_{k}\right)\right]=\rho\left[x_{0}, f\left(x_{0}, 0\right)\right]=\rho\left[x_{0}, x_{0}\right]=0$, which is a contradiction. In a similar manner it may be shown that $\lim _{n \rightarrow \infty} \max _{x \in \varepsilon} \rho\left[x, h_{n}^{\prime \prime}(x)\right]$ $=0$. Consequently the sequences $\left\{S_{n}^{\prime}\right\}_{1}^{\infty}$ and $\left\{S_{n}^{\prime \prime}\right\}_{1}^{\infty}$ of closed 2-manifolds in $S^{3}$ converge homeomorphically to $S$ from opposite sides. It therefore follows from Bing's characterization of tame surfaces that $S$ is tame in $S^{3}$ [5].

The following extension of Theorem 5 will be needed in the proof of Theorem 12.

Theorem 11. Suppose that $A, B$, and $C$ are solid tori such that $A \subset \operatorname{Int} B$ and $B C \operatorname{Int} C$, with $\mathrm{Bd} B$ collared in $C$. Then $A$ and $C$ are concentric if and only if $B$ is concentric with both $A$ and $C$.

Proof. First suppose that $A$ and $C$ are concentric. Let $D$ be a solid torus and suppose that the boundary of $D$ is identified with the boundary of $C$ in such a way that $C \cup D$ is the 3 -sphere $S^{3}$. Let $E$ be a solid torus interior to $D$ and concentric with $D$, and let $F$ be a solid torus interior to $A$ and concentric with $A$. Then $\mathrm{Cl}(D-E)$ is the topological product of a torus and an interval so that there is a homeomorphism $f$ of $\mathrm{Bd} C \times[0,1]$ onto $\mathrm{Cl}(D-E)$ such that $f(x, 0)=x$ for each $x \in \mathrm{Bd} C$, and $\mathrm{Cl}(C-A)$ is the topological product of a torus and an interval so that there is a homeomorphism $g$ of $\mathrm{Bd} C \times[-1,0]$ onto $\mathrm{Cl}(C-A)$ such that $g(x, 0)=x$ for each $x \in \mathrm{Bd} C$. If the

(2) The author is indebted to the referee for helpful suggestions in connection with this section. 
map $h$ of $\mathrm{Bd} C \times[-1,1]$ onto $\mathrm{Cl}(C-A) \cup \mathrm{Cl}(D-E)$ is defined by $h(x, t)$ $=g(x, t)$ if $t \in[-1,0]$ and $h(x, t)=f(x, t)$ if $t \in[0,1]$, then $h$ is clearly a homeomorphism such that $h(x, 0)=x$ for $x \in \mathrm{Bd} C$, so that $\mathrm{Cl}(C-A)$ $\cup \mathrm{Cl}(D-E)$ is a collar of $\mathrm{Bd} C$ in $S^{3}$. Similarly $\mathrm{Cl}(C-A) \cup \mathrm{Cl}(A-F)$ is a collar of $\mathrm{Bd} A$. It therefore follows from Lemma 4 that the solid tori $A, B$, and $C$ are tame in $S^{3}$. Consequently Theorem 5 now applies to show that $B$ is concentric with both $A$ and $C$.

Conversely, if $B$ is concentric with both $A$ and $C$, then it may be shown as above that $\mathrm{Cl}(C-A)=\mathrm{Cl}(C-B) \cup \mathrm{Cl}(B-A)$ is a collar of $\mathrm{Bd} B$, so that $A$ and $C$ are concentric.

By a closed 3-manifold is meant a compact connected separable metric space, each point of which has a neighborhood which is homeomorphic to Euclidean 3-space.

THEOREM 12. Let $M$ be a closed 3-manifold and suppose that $\mathcal{H C}$ is an ununcountable collection of solid tori in $M$, whose boundaries are mutually disjoint and are collared in $M$. Then ${ }^{3 C}$ contains a pair of concentric solid tori.

Proof. Notice first that if $B$ is an element of $\mathfrak{H}$, then the boundary $T$ of $B$ separates $M$. For if Int $B$ denotes the set of those points of $B$, each of which has a neighborhood in $B$ which is homeomorphic to $E^{3}$, then it follows from Brouwer's theorem on the invariance of interior points $[1, \mathrm{p} .168]$ that Int $B=B-T$ is an open subset of $M$. Since $B$ is compact, $M-B$ is also an open subset of $M$. Then $M-T$ is the union of the two disjoint open sets Int $B$ and $M-B$, so that $M-T$ is not connected.

Suppose that $\mathfrak{H C}$ is indexed by an uncountable set $\Lambda$, so that $\mathfrak{H C}=\left\{B_{\alpha}: \alpha \in \Lambda\right\}$. For each $\alpha \in \Lambda$, let $T_{\alpha}=\operatorname{Bd} B_{\alpha}$. Since $M$ is separable, let $D$ be a countable dense subset of $M$, and define a mapping $\phi$ of $\mathcal{F}$ into $D \times D$ by associating with the element $B_{\alpha}$ of $\mathfrak{F C}$ an element $\phi\left(B_{\alpha}\right)=(x, y)$ of $D \times D$ such that $T_{\alpha}=\operatorname{Bd} B_{\alpha}$ separates $x$ and $y$ in $M$, with $x \in \operatorname{Int} B_{\alpha}$ and $y \in M-B_{\alpha}$. Since $\mathcal{F C}$ is uncountable and $D \times D$ is countable, it follows that there is an element $(a, b)$ of $D \times D$ such that the subcollection $\mathcal{F}^{\prime}=\phi^{-1}(a, b)$ of $\mathcal{H C}$ is uncountable. Thus $\mathfrak{H C}^{\prime}$ is an uncountable subcollection of $\mathfrak{H C}$ such that, given $B_{\alpha} \in \mathcal{F C}^{\prime}$, the two points $a$ and $b$ of $M$ are separated by the boundary $T_{\alpha}$ of $B_{\alpha}$, with $a \in$ Int $B_{\alpha}$ and $b \in M-B_{\alpha}$.

Now suppose that $B_{\alpha}$ and $B_{\beta}$ are any two solid tori in $\mathcal{F C}^{\prime}$. Since $T_{\alpha} \cap T_{\beta}$ $=\square$, either $T_{\alpha} \subset$ Int $B_{\beta}$ or $T_{\alpha} \subset M-B_{\beta}$. If $T_{\alpha} \subset$ Int $B_{\beta}$, then $B_{\alpha} \subset$ Int $B_{\beta}$. To the contrary, suppose that $z \in \operatorname{Int} B_{\alpha}-\operatorname{Int} B_{\beta}$. Since $a, z \in \operatorname{Int} B_{\alpha}$, there is an arc $l_{1}$ joining $a$ and $z$ and not meeting $T_{\alpha}$. Since $b, z \in M-\operatorname{Int} B_{\beta}$ and $T_{\alpha} \subset$ Int $B_{\beta}$, there is an arc $l_{2}$ joining $b$ and $z$ and also not meeting $T_{\alpha}$. But then $l_{1} \cup l_{2}$ is a connected subset of $M-T_{\alpha}$ which contains the points $a$ and $b$, and this is a contradiction. Similarly $T_{\alpha} \subset M-B_{\beta}$ implies that $B_{\beta} \subset \operatorname{lnt} B_{\alpha}$. Hence $\mathcal{F C}^{\prime}$ is an uncountable subcollection of $\mathfrak{F}$ such that, if $B_{\alpha}$ and $B_{\beta}$ are 
any two solid tori in $\mathrm{JC}^{\prime}$, either $B_{\alpha} \subset$ Int $B_{\beta}$ or $B_{\beta} \subset$ Int $B_{\alpha}$. It may therefore be assumed that the original collection $\mathfrak{H}$ has this property.

Now Whyburn [12] has shown that every uncountable nonseparated collection $\mathcal{G}$ of cuttings of a connected separable metric space $M$ contains an uncountable subcollection $\overline{\mathcal{G}}$, such that to each element $T$ of $\overline{\mathcal{G}}$ and each point $p$ of $M-T$ there corresponds an element $T^{\prime}$ of $\overline{\mathcal{G}}$ which separates $T$ and $p$ in $M$. Applying this theorem with $\mathcal{G}$ being the collection of boundaries of the solid tori in $\mathcal{H}$, let $T_{\alpha_{0}}$ be an element of $\overline{\mathcal{G}}$, with $B_{\alpha_{0}}$ the corresponding solid torus bounded by $T_{\alpha_{0}}$. Since $T_{\alpha_{0}}$ is by hypothesis collared in $M$, there is a homeomorphism $f$ of $T_{\alpha_{0}} \times[0,1]$ into $M-\operatorname{Int} B_{\alpha_{0}}$ such that $f(x, 0)=x$ if $x \in T_{\alpha_{0} .}$ Then $B_{\alpha_{0}} \cup f\left(T_{\alpha_{0}} \times[0,1]\right)$ is a solid torus $C$ containing $B_{\alpha_{0}}$ in its interior and concentric with $B_{\alpha_{0}}$. Then, given any point $x \in \mathrm{Bd} C$, there is a torus $T_{\alpha_{x}}$ in $\overline{\mathcal{G}}$ separating $x$ and $T_{\alpha_{0}}$ in $M$. Clearly $B_{\alpha_{0}} \subset \operatorname{Int} B_{\alpha_{x}}$, so that the collection $\left\{M-B_{\alpha_{x}}\right\}_{x \in \mathrm{Bd} C}$ of open sets covers Bd $C$. Since Bd $C$ is compact, there is a finite subcollection $\left\{M-B_{\alpha_{n}}\right\}_{n=1}^{k}$ which covers $\mathrm{Bd} C$. Since either $B_{\alpha} \subset$ Int $B_{\beta}$ or $B_{\beta} \subset$ Int $B_{\alpha}$ for every $\alpha, \beta \in \Lambda$, it may be assumed that $\mathrm{Bd} C \subset M$ $-B_{\alpha_{1}}$, so that $T_{\alpha_{1}}$ separates $T_{\alpha_{0}}$ and $\mathrm{Bd} C$ in $M$. It follows that $B_{\alpha_{1}} C$ Int $C$. For otherwise, if there were a point $p \in \operatorname{Int} B_{\alpha_{1}}-\operatorname{Int} C, p$ could be joined by an arc not meeting $T_{\alpha_{1}}$ to a point $x \in T_{\alpha_{0}}$ because $T_{\alpha_{0}} \subset$ Int $B_{\alpha_{1}}$, and $p$ could be joined by an arc not meeting $T_{\alpha_{1}}$ to a point $y \in \mathrm{Bd} C$ because $T_{\alpha_{1}} \subset \operatorname{Int} C$. Since therefore $B_{\alpha_{0}} \subset$ Int $B_{\alpha_{1}}$ and $B_{\alpha_{1}} \subset$ Int $C$, and since it may be assumed that the collar of $T_{\alpha_{1}}$ is contained by $C$, Theorem 11 applies to show that $B_{\alpha_{0}}$ and $B_{\alpha_{1}}$ are concentric. Consequently $\mathcal{H}$ contains pairs of concentric solid tori.

CoRollary 6. If $M$ and $\mathcal{H C}$ are as in Theorem 12 , then $\mathcal{H}$ contains an uncountable subcollection $\mathrm{HC}^{*}$ such that any two solid tori in $\mathrm{HC}^{*}$ are concentric.

Proof. Define $B_{\alpha} \sim B_{\beta}$ if either $B_{\alpha}=B_{\beta}$ or the two solid tori $B_{\alpha}$ and $B_{\beta}$ are concentric. Since it may be assumed, as shown in the proof of Theorem 12, that, given $B_{\alpha}$ and $B_{\beta}$ in $\mathcal{H}$, either $B_{\alpha} \subset$ Int $B_{\beta}$ or $B_{\beta} \subset$ Int $B_{\alpha}$, it follows from Theorem 11 that this relation is an equivalence relation in $\mathcal{H}$. There is thereby induced a decomposition of $\mathfrak{H C}$ into mutually disjoint equivalence classes, such that two solid tori in $\mathcal{H C}$ are elements of the same equivalence class if and only if they are concentric. An application of the axiom of choice gives a collection $\mathscr{K}$ containing exactly one element of each equivalence class. Since no two solid tori in $\mathcal{K}$ are concentric, it follows from Theorem 12 that $\Re$ must be countable. Therefore one of the equivalence classes must be uncountable. If $\mathfrak{H}^{*}$ denotes this uncountable equivalence class, then $\mathfrak{H}^{*}$ is an uncountable subcollection of $\mathcal{H}$ such that any two solid tori in $\mathcal{F}^{*}$ are concentric.

THEOREM 13. If $\mathcal{G}$ is an uncountable collection of mutually disjoint tori in the closed 3-manifold $M$, then $\mathrm{G}$ contains an uncountable subcollection $\mathcal{G}^{*}$ such that any two tori in $\mathrm{G}^{*}$ are concentric in $M$. 
Proof. There are two obstructions to an immediate application of Theorem 12 or Corollary 6 . First, the elements of $\mathcal{G}$ may not bound solid tori in $M$, and second, the tori in $\mathcal{G}$ may not be collared in $M$. However the latter is no real difficulty, since, using results of Bing [4], it may be assumed that each of the tori in $\mathcal{G}$ is tame, and hence is collared in $M$. The first difficulty will be circumvented by finding a torus $T_{0}$ in $\mathcal{G}$ whose collar $C\left(T_{0}\right)$ contains uncountably many elements of $\mathcal{G}$, so that a simplification to the case of a 3sphere can be achieved by sewing on two solid tori along the boundary of $C\left(T_{0}\right)$.

Since the 2-dimensional connectivity number (using modulo 2 coefficients) of the closed 3-manifold $M$ is finite, there is a non-negative integer $n$ such that $\mathcal{G}$ contains $n$ elements whose union does not separate $M$, while the union of any $n+1$ elements of $g$ does separate $M$. Therefore let $T_{1}, \cdots, T_{n}$ be elements of $S$ such that $M-\left(T_{1} \cup \ldots \cup T_{n}\right)$ is connected, while $M-\left(T_{1} \cup \ldots \cup T_{n} \cup T\right)$ is not connected if $T$ is an element of $\mathcal{G}-\left\{T_{1}, \cdots, T_{n}\right\}$. Assuming that each of the tori $T_{i}, i=1, \cdots, n$, has a collar $C\left(T_{i}\right)$ not containing uncountably many elements of $\mathcal{G}$, let $\bar{M}$ be the compact 3-manifold obtained by cutting $M$ along $T_{1}, \cdots, T_{n}$, or $\bar{M}=\mathrm{M}$ $-\bigcup_{i=1}^{n}$ Int $C\left(T_{i}\right)$, with the collars $C\left(T_{i}\right)$ chosen mutually disjoint $(\bar{M}=M$ if $n=0)$. It is now clear that $\bar{M}$ is separated by each torus of the uncountable subcollection $\overline{\mathcal{G}}$ consisting of those elements of $\mathcal{G}$ which are contained in Int $\bar{M}$. Consequently Whyburn's results [12] on uncountable nonseparated collections of cuttings of a connected separable metric space can be employed to find an uncountable subcollection $\mathcal{G}^{\prime}$ of $\overline{\mathcal{G}}$ and an upper semicontinuous decomposition space $M^{\prime}$ of $\bar{M}$ in which the elements of $\mathcal{G}^{\prime}$ are points. If the element $T_{0}$ of $\mathcal{G}^{\prime}$ is a condensation point of $\mathcal{G}^{\prime}$ in $M^{\prime}$, then the collar $C\left(T_{0}\right)$ of $T_{0}$ in $M$ contains an uncountable subcollection $\mathcal{G}^{\prime \prime}$ of the elements of $\mathcal{G}$. Now identify the boundaries of two solid tori $B_{1}$ and $B_{2}$ with the boundary components of $C\left(T_{0}\right)$ in such a way that $B_{1} \cup C\left(T_{0}\right) \cup B_{2}$ is the 3 -sphere $S^{3}$. By a theorem of Alexander [2], there may be assigned to each torus $T_{\alpha}$ in $\mathcal{G}^{\prime \prime}$ a solid torus $B_{\alpha}$ in $S^{3}$ bounded by $T_{\alpha}$, with $B_{\alpha}$ containing either $B_{1}$ or $B_{2}$ in its interior (since it may be assumed without loss that each element of $\mathcal{G}^{\prime \prime}$ separates the boundary components of $C\left(T_{0}\right)$ ). Corollary 6 now applies to show that $\mathcal{G}^{\prime \prime}$ contains an uncountable subcollection $\mathcal{G}^{*}$ such that any two tori in $\mathrm{G}^{*}$ are concentric in $C\left(T_{0}\right)$ and therefore in $M$.

\section{BIBLIOGRAPHY}

1. P. S. Aleksandrov, Combinatorial topology, Vol. 1, Graylock Press, Rochester, N. Y., 1956.

2. J. W. Alexander, On the sub-division of space by a polyhedron, Proc. Nat. Acad. Sci. U.S.A. 10 (1924), 6-8.

3. R. H. Bing, Locally tame sets are tame, Ann. of Math. 59 (1954), 145-158.

4. $-E^{3}$ does not contain uncountably many mutually exclusive wild surfaces, Abstract 63-801t, Bull. Amer. Math. Soc. 63 (1957), 404. 
5. - Conditions under which a surface in $E^{3}$ is tame, Fund. Math. 47 (1959), 105-139.

6. C. H. Edwards, Concentric tori in the three-sphere, doctoral dissertation, University of Tennessee, December, 1960.

7. - Concentric tori in the 3-sphere, Bull. Amer. Math. Soc. 67 (1961), 220-222.

8. - A characterization of tame curves in the 3-sphere, Abstract 573-32, Notices Amer. Math. Soc. 7 (1960), 875.

9. O. G. Harrold, H. C. Griffith, and E. E. Posey, A characterization of tame curves in 3-space, Trans. Amer. Math. Soc. 79 (1955), 12-35.

10. H. Schubert, Knoten und Vollringe, Acta Math. 90 (1953), 132-286.

11. - Die eindeutige Zerlegbarkeit eines Knotens in Primknoten, S.-B. Heidelberger Akad. Wiss. Math. Nat. K1. 1949, no. 3 (1949), 57-104.

12. G. T. Whyburn, Non-separated cuttings of connected point sets, Trans. Amer. Math. Soc. 33 (1931), 444-454.

The University of Tennessee,

KNOXVILLE, TeNNESSEE 